\section{Las tendencias como estrategia de innovación en el diseño de productos sostenibles para niñxs}

Beatriz García Prósper y Patricia

Rodrigo Franco ${ }^{(1)}$

Resumen: La creciente preocupación por el cambio climático en el público infantil, manifiesta a través del activismo global, genera una demanda a la que los diseñadores deben dar respuesta. El estudio presenta una herramienta para creativos, basada en el análisis de tendencias, que permite desarrollar nuevos productos sostenibles.

La herramienta se fundamenta en la observación de estilos de vida, casos de empresas y tendencias de diseño cuya metodología aporta claves para proponer briefings innovadores de productos sostenibles. Un instrumento de código visual que ofrece información sobre el comportamiento del consumidor y permite conocer con antelación productos y estéticas que estarán vigentes, facilitando una dirección estratégica del diseño.

Palabras clave: infantil - activismo - tendencias - sostenible - observación - diseño - innovadores - estrategia.

[Resúmenes en inglés y portugués en la página 83]

(1) Escuela Técnica Superior de Ingeniería del Diseño, Universitat Politècnica de València, España.

\title{
Introducción
}

No hay vuelta atrás. El desarrollo de productos sostenibles es una demanda creciente que los diseñadores deben abordar cuanto antes. El cambio climático, como cuestión sensible en el público infantil, ha derivado en un activismo global liderado por los seres humanos mas jóvenes del planeta.

Según datos de Open Science Framework ${ }^{1}$, en septiembre de 2019, la tercera huelga climática global organizada por la campaña de protesta Fridays For Future movilizó 6000 eventos de protesta en 185 países y sacó a las calles a 7,6 millones de participantes en su mayoría niños y niñas.

Por otro lado, la investigación en diseño, centrada en la innovación, ha generado estudios de diversa índole entre los que se encuentran los análisis de prospectiva y tendencias. 
Aplicar al proceso de diseño metodologías y técnicas propias de los análisis de tendencias, proporciona a los diseñadores la capacidad de visualizar con mayor claridad las futuras líneas de acción a emprender en cualquier contexto.

En este artículo se propone una herramienta basada en la observación de estilos de vida y tendencias infantiles centradas en el contexto de la sostenibilidad. Un instrumento de trabajo para que creadores y diseñadores aborden proyectos que den respuesta a esta preocupación.

Sostenibilidad es avanzar en armonía con nosotros mismos, los demás y la naturaleza. El concepto de sostenibilidad aparece por primera vez en el Informe Brundtland ${ }^{2}$ de 1987. En dicho informe se alertó por primera vez sobre las consecuencias medioambientales negativas del desarrollo económico y la globalización, tratando de ofrecer soluciones a los problemas derivados de la industrialización y el crecimiento poblacional. Desde aquella primera advertencia, la situación ha empeorado considerablemente, provocando una reacción sobretodo en las generaciones más jóvenes que ven como se deteriora el planeta que van a heredar.

Los estudios de consumo elaborados por expertos en diversos contextos reflejan precisamente la sensibilización con el problema en una determinada franja de edad que en la mayoría de los casos no supera la treintena. En este sentido, en un entorno sensible a esta cuestión cómo es la alimentación, se evidencia como los consumidores menores de 35 años son los mas numerosos en cuanto a consumo de alimentos 'bio' según el Ministerio de Agricultura ${ }^{3}$ español. Esta creciente demanda demuestra una tendencia hacia la sensibilización de las generaciones de nuevos padres y madres sobre la sostenibilidad.

Paralelamente, como indica una reciente investigación desarrollada por $\mathrm{AIJU}^{4}$ sobre el consumo de juguetes ecológicos, el $21 \%$ de las familias tiene en cuenta la sostenibilidad a la hora de adquirir juguetes, porcentaje que aumenta hasta $31 \%$ en familias jóvenes con padres y madres millenial y con hijos pequeños. El 63\% de las familias consideran que la oferta de juguetes sostenibles es baja o muy baja. Y la mitad de las familias piensa que en los próximos 5 años aumentará la preocupación de la sociedad en torno a los juguetes sostenibles.

El estudio realizado sobre 3000 familias en España durante la campaña de navidad 20192020, sostiene que la preocupación sobre el consumo sostenible aumenta paulatinamente. De hecho, la comparativa entre las campañas 2018/2019 vs 2019/2020 refleja un aumento del comprador ecofriendly que pasa del $9 \%$ al $16 \%$, un aumento del comprador sensible del $21 \%$ al $26 \%$ y una disminución del comprador indiferente que pasa del $70 \%$ al $58 \%$. Estos datos revelan una clara tendencia hacia la conciencia sobre el consumo sostenible de productos infantiles. Además, según la investigación realizada, es un hecho que los compradores actuales están más informados; buscan más datos sobre los juguetes que compran y compran menos plástico y más juguetes de madera. Por último, creen que la sostenibilidad beneficia la salud de todas y todos.

Ante esta nueva realidad, los diseñadores deben dirigir sus estrategias creativas en una dirección clara: generar productos coherentes con la protección del planeta, que aseguren un mundo habitable a las futuras generaciones. 


\section{Objetivos}

Los diseñadores deben abordar con urgencia el desarrollo de productos infantiles sostenibles que ayuden a mitigar el cambio climático.

Para dar respuesta a esta demanda creciente, se propone una herramienta de trabajo basada en la observación de tendencias y estilos de vida sostenibles en la infancia. La herramienta ofrece una información que permite el conocimiento del contexto y facilita a los creativos diseñar dichos productos.

Conocer productos, actitudes y estéticas acordes al estilo de vida sostenible, requiere la obtención de información en base a tres parámetros importantes.

En primer lugar, se pretende abordar la fase previa de exploración del entorno, habitual en cualquier proceso de diseño, partiendo de información relativa a productos, servicios y empresas creadoras de tendencias con una clara dirección sostenible.

Por otro lado, se plantea como objetivo empatizar con el usuario potencial, afín y sensible a la protección del medio ambiente, a través de la observación en el plano sociocultural de actitudes y estilos de vida comprometidos con la sostenibilidad.

En tercer lugar, atendiendo a la índole práctica del diseño, se pretende identificar cuáles son los acabados (materiales, colores, gráficas y texturas) que se identifican como sostenibles. En definitiva, el objetivo principal es proponer briefings innovadores de productos sostenibles. El método que se propone para conseguirlo es la utilización de la herramienta de 'tendencias de diseño'.

\section{Metodología}

La herramienta está basada en el método analítico ${ }^{5}$ de las tendencias de diseño y los servicios de innovación. La metodología de análisis de las tendencias se basa en un sistema de vigilancia de fuentes de información fundamentado en la detección, el rastreo y la observación de comportamientos sociales, puntos de venta emblemáticos, ferias internacionales de alto impacto y revisión online en el contexto del diseño infantil. El planteamiento de las tendencias es una herramienta que permite a los creativos diseñar productos innovadores con una visión prospectiva.

Esta herramienta se presenta bajo el formato del cuaderno de tendencias Kids Trends $B o o k^{6}$. Se trata de un documento que aglutina de forma gráfica las orientaciones desde la perspectiva de cuatro tendencias. De modo que el cuaderno funciona como un instrumento de inspiración para equipos creativos que interpretan las tendencias, lo que les permite desarrollar nuevas líneas de producto atendiendo a las orientaciones presentadas. El cuaderno de tendencias Kids Trends Book 2022 plantea cuatro estilos de vida infantiles: Good Vibes Planet ${ }^{7}$, Big Gang Theory ${ }^{8}$, Tra-Trá! ${ }^{9}$ y, por último, Honest Activism ${ }^{10}$. Cada una de las tendencias aborda distintos estilos de vida de usuarios y usuarias infantiles. Los niños y niñas se comportan como cualquier otro consumidor relacionándose con productos y servicios acordes a su modo de entender la vida. De los cuatro estilos de vida, que 
configuran el cuaderno, la propuesta que se encuentra directamente relacionada con la preocupación por el cambio climático en el público infantil es 'Honest Activism'.

A través de la información derivada de la propuesta de esta tendencia se desarrolla el presente estudio. La estructura de la herramienta nos aporta datos cualitativos sobre indicadores, iconos y señales. Cada uno de estos apartados aborda propuestas que dan respuesta a las inquietudes del colectivo que representan.

\section{Indicadores}

Los indicadores son las actitudes mas representativas de la tendencia que se ilustran a través de actividades propuestas por colectivos sensibles. Se plantean cinco indicadores:

1. Movilizando conciencia. Luchando por el futuro: Fridays for Future ${ }^{11}$

2. Nutrición colaborativa. Aprendiendo cocina sostenible: Programme bote à lunch ${ }^{12}$

3. Derecho a aprender para todxs. Educando en la igualdad: Peggy Notebaert Nature Museum $^{13}$

4. Solidarios por naturaleza. Pescando basura del mar: Ocean Guardians Assemble ${ }^{14}$

5. Comprometidos con la sociedad. Sentando las bases para el respeto: Human Rights Reads for Kids ${ }^{15}$

\section{Iconos}

Los iconos son las personas que lideran, culturalmente hablando, el estilo de vida representado. El colectivo que aglutina esta tendencia se identifica con valores y actitudes representados por estos prescriptores. Kids Trends Book plantea cinco iconos:

1. Greta Thunberg ${ }^{16}$.

2. Chloé Sucrée ${ }^{17}$.

3. Malala Yousafzai ${ }^{18}$.

4. Fionn Ferreira ${ }^{19}$.

5. Mary Copeny ${ }^{20}$.

\section{Señales}

Esta fase aborda el conjunto de información necesaria, en cualquier proyecto de diseño, durante las etapas de investigación y de documentación previas a las de bocetado.

Señal 1. Hábitat: Nido Muebles con 'Traven'21

Señal 2. Juguetes: Plan Toys con 'Wonky Fruits'22

Señal 3. Gráfica: Bobo Choses con 'We Cosmos Collection'23

Señal 4. Estilo de vida: Little Eco Baby con 'pañales lavables’24

El comportamiento del consumidor se aborda a través de los 'indicadores' aportando datos sobre actitudes, valores y estilos de vida que se relacionan con el diseño y las influencias. Y, siguiendo la metodología etnográfica ${ }^{25}$ de análisis de tendencias, el estudio se completa con la localización de 'iconos' referentes en el estilo de vida objeto de estudio. Una vez estudiados los argumentos de la tendencia se deben traducir en productos orientados 
al futuro y al consumidor en un mercado concreto. $Y$ es aquí donde las 'señales' adquieren valor proyectual. De hecho, la herramienta Kids Trends Book facilita una paleta cromática y de materiales que funcionan como guía de estilo de la que partir para el desarrollo ulterior dependiendo del tipo de proyecto.

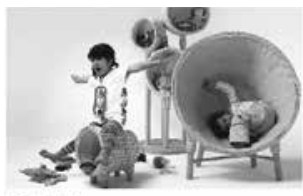

Señal 1.

Nido Muebles

'Traven'

1

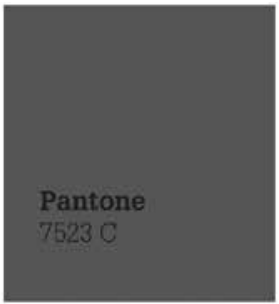

2
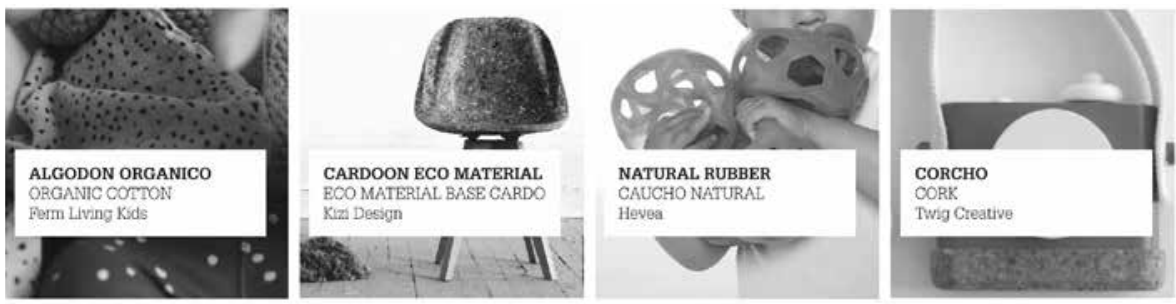

3

Figura 1. Señales de Kids Trends Book 2022. Figura 2. Paleta de color de Kids Trends Book 2022. Figura 3. Paleta de materiales de Kids Trends Book.

\section{Laboratorio proyectual}

Con el objetivo de testar la herramienta KidsTrends Book se plantea un laboratorio proyectual con jóvenes diseñadores formados en la Universitat Politècnica de València. 
La experiencia contempla distintas fases. En primer lugar, se plantean unas premisas basadas en criterios de sostenibilidad. Estos parámetros son los siguientes:

- Respeta el medio ambiente: uso mínimo de materiales diferentes. Promover actitudes y acciones de cuidado del entorno natural.

- Durabilidad: generar productos duraderos que se puedan reaprovechar o transformar. Eliminar la idea de residuo.

- Fin de vida: biodegradable. Fácil de desensamblar. Separar para reciclar.

- Sensibilización social: conciencia y empatía con el medio ambiente. Mejorar la situación de los espacios y de las personas.

- Impacto positivo: crear valor a largo plazo. No cargar con el mantenimiento o las consecuencias del producto en el futuro.

A continuación, se ofrece la herramienta Kids Trends Book. A partir de su análisis, se aborda el proceso de ideación. El instrumental proporcionado, permite a los diseñadores empatizar con los usuarios, intentando satisfacer necesidades concretas y aplicando las conclusiones derivadas de las tendencias a ideas de productos y negocios sostenibles.

El proceso metodológico propuesto para el desarrollo de nuevos productos enfatiza las primeras fases dedicadas a la investigación de los entornos internos y externos a la empresa. En este caso, se evidencia la importancia del análisis de los entornos externos, referidos a factores de tipo sociocultural, donde se generan las definiciones de los estilos de vida y su identificación con tendencias concretas. Es aquí donde se plantean el mayor número de oportunidades para el diseño de nuevos productos.

A continuación, se muestran tres de los productos resultantes en dicho experimento.

\section{Resultados}

La aplicación de la herramienta permite diseñar productos centrados en dar respuesta a las nuevas demandas de la sociedad en materia de sostenibilidad.

Como se ha visto con anterioridad esta metodología ofrece parámetros que permiten a los diseñadores plantear productos innovadores en tres direcciones. Dentro del contexto productivo se proporciona información relativa a productos y empresas con una clara dirección sostenible. Este planteamiento facilita a los creativos abordar la fase previa de exploración de productos habitual en cualquier proceso de diseño. El interés reside en que los casos planteados son creadores de tendencias ${ }^{26}$. A partir de estos referentes el creativo puede seguir indagando en su estudio personalizado dependiendo del producto objeto de diseño.

Paralelamente, la herramienta ofrece información sobre el contexto social que es afín y sensible al medio ambiente. Se trata de personas activas y que son referente en esta tendencia sostenible. También, se aporta información sobre actividades y acciones que proporcionan indicadores del estilo de vida de este colectivo. Contar con estos referentes ayuda al diseñador a empatizar con sus usuarios potenciales. 
En tercer lugar, la herramienta proporciona información concreta a modo de guía de diseño. En esta ocasión, se ofrece información de índole práctica y aplicada sobre acabados identificables como sostenibles, concretamente: paleta de color y guía de materiales. Disponer de datos precisos sobre las terminaciones de los productos y su estética ayudan considerablemente a proponer y comunicar los productos de manera acorde al estilo de vida estudiado.

En base a estos tres parámetros se aportan a continuación los resultados de la experiencia de uso de la herramienta.

El contexto productivo determina el ecosistema en el que los productos diseñados van a convivir. La información sobre los casos de productos, servicios y empresas aporta conocimiento para idear briefings innovadores de productos en sintonía con dicho contexto. A partir de las señales, que plantea la herramienta, se establece el diseño conceptual.

- Concepto Producto $1^{27}$ : Juego outdoor, activo y de rol.

- Concepto Producto $2^{28}$ : Juego creativo y manipulativo.

- Concepto Producto $3^{29}$ : Juego de mesa.
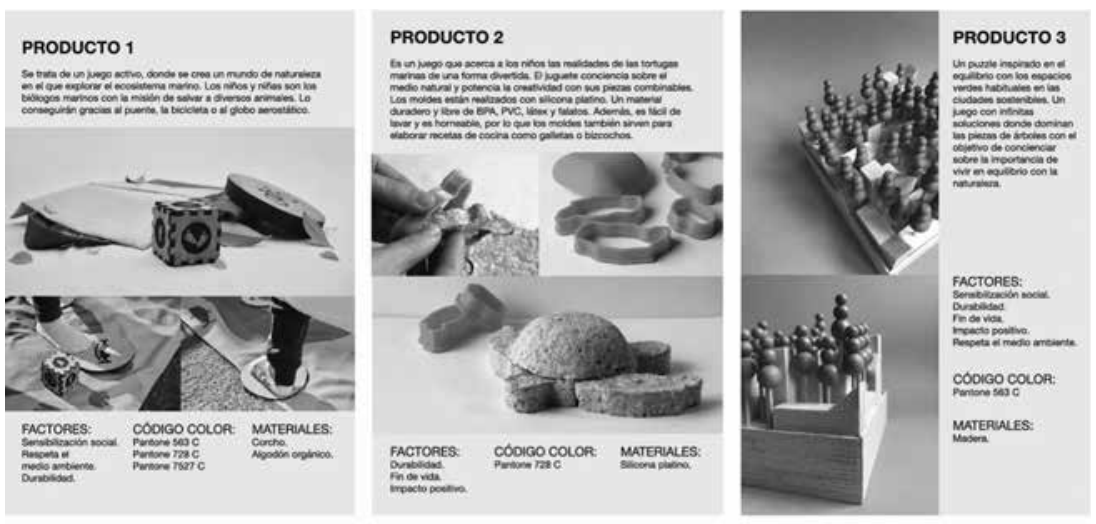

Figura 4. Guía de diseño.

El contexto social es el segundo parámetro donde se evidencian los factores que ofrece el producto en base a los indicadores de la tendencia. A través de los estilos de vida observados se establecen los principios de sostenibilidad que deben abordar los productos diseñados: respeta el medio ambiente, durabilidad, fin de vida, sensibilización social e impacto positivo.

Los productos 1 y 2 , con su planteamiento, abordan la sensibilización hacia la preservación de los ecosistemas marinos. El producto 3 se centra en el equilibrio entre zonas 
verdes y urbanas de las ciudades sostenibles. Los tres productos están concebidos desde la premisa de los principios de sostenibilidad fruto del contexto social.

- Factores Producto 1: sensibilización social, respeta el medio ambiente y durabilidad.

- Factores Producto 2: durabilidad, fin de vida e impacto positivo.

- Factores Producto 3: sensibilización social, durabilidad, fin de vida, impacto positivo y respeta el medio ambiente.

El tercer parámetro aplicado en los tres productos responde a la guía de diseño. Los códigos de color utilizados parten de las recomendaciones que la herramienta propone como punto de partida. Lo mismo ocurre en el caso de los materiales, que surgen a partir de las propuestas indicadas adaptándose a cada producto concreto. Por tanto, se trata de interpretar las indicaciones sobre acabados que ofrece la herramienta que aporta sugerencias para elaborar una guía de estilo propia.

- Guía de diseño Producto 1: parte de una propuesta de color condicionada por el material de corcho. La gama se amplia con colores fríos aplicados al tapiz de algodón orgánico, generando contraste entre las piezas.

- Guía de diseño Producto 2: a partir del estudio de materiales flexibles como el caucho surgen nuevas iniciativas que aseguran un impacto positivo en el medio ambiente y se adaptan a los diferentes usos del juguete. El código de color responde al material.

- Guía de diseño Producto 3: Parte de un código de color que simboliza los dos elementos que se enfrentan en el juego. Por un lado, los edificios representados por la tonalidad neutra de la madera sin tratar y, por otro lado, el color verde de los árboles.

\section{Conclusiones}

Con la herramienta de observación y análisis Kids Trends Book se detectan las señales que pronostican las tendencias. La labor instrumental de reconocer los cambios que se producen en el entorno y descifrarlos en clave de briefings innovadores favorece la toma de decisiones estratégicas para creativos y fortalece su visión de futuro e innovación.

Las agencias internacionales de tendencias como abordan pronósticos que incluyen, en mayor o menor medida, observaciones sobre el mundo infantil. En este contexto Kids Trends Book es el único caso que aborda diferentes sectores infantiles especialmente el del juguete.

Durante el presente trabajo se ha expuesto la aplicación de la herramienta KidsTrends Book como alternativa metodológica al diseño de productos sostenibles en el ámbito infantil. Esta tipología de trabajo aborda cuestiones que aportan un valor metodológico a los procesos tradicionales. Los teóricos del diseño han reflexionado sobre la metodología proyectual a lo largo de la historia. En este sentido, Bruno Munari ${ }^{30}$ la describía como una fórmula de doce pasos esenciales para implementar un método que ayudara a resolver un 
problema desde su planteamiento inicial hasta la respuesta final, atravesando una serie de fases establecidas que le conducirían a encontrar una solución adecuada.

En la actualidad, los planteamientos metodológicos ${ }^{31}$ de innovación ágil, por ejemplo, vienen dando respuesta a las técnicas tradicionales de diseño proponiendo valores de los que se nutren las metodologías ágiles. Estos métodos, que nacieron para el desarrollo ágil de software, se han ido adaptando a otras áreas como el diseño de producto. Las metodologías ágiles ${ }^{32}$ son muy utilizadas en los procesos de generación de nuevas ideas que permiten implementar procesos de innovación; desarrollar nuevos productos y reconocer oportunidades de mercado.

Desde esta perspectiva, el Kids Trends Book aporta una herramienta para visualizar tendencias que ofrece información relevante sobre el comportamiento del consumidor y permite conocer qué productos y estéticas sostenibles van a estar vigentes y marcan tendencia. Desde el punto de vista práctico, es un instrumento para la toma de decisiones que permite plantear briefings innovadores y evaluar propuestas para nuevos productos sostenibles. Como guía de comunicación ilustra conceptos abstractos y ayuda a entenderlos agilizando el proceso de comunicación de ideas y poniendo en sintonía a los equipos de marketing y diseño.

Trabajar esta metodología, como guía de producto sostenible, facilita una dirección estratégica del diseño que permite mejorar la colección, posicionar la marca y anticiparse a sus competidoras. En el contexto de los materiales, la herramienta proporciona una visión estratégica donde descubrir nuevos materiales sostenibles; rescatar nuevas aplicaciones de componentes tradicionales o afianzar aquellos que persisten en el tiempo. Del mismo modo, el manual permite obtener una visión actualizada del color y los estilos gráficos contemporáneos relacionados con la idea de sostenibilidad.

Abordar procesos de diseño desde el punto de vista de la sostenibilidad es una exigencia ineludible que los creativos deben incorporar de inmediato. Los diseñadores han participado en todos los grandes desafíos a los que la humanidad se ha enfrentado con propuestas que han facilitado la adaptación al cambio. ¿Por qué iba a ser ahora distinto?

\section{Notas}

1. AAVV. (2020, 23 de febrero). Surveys of participants in Fridays For Future climate protests on 20-28 September, 2019, in 19 cities around the world. Medio. https://osf.io/asruw/ 2. World Commission on Environment and Development (1987). Our Common Future: Brundtland Report. Oxford University Press.

3. Ministerio de Agricultura y Pesca, Alimentación y Medio Ambiente (2017, julio). Caracterización de compradores de productos ecológicos en canal especializado. Secretaría General Técnica Centro de Publicaciones Medio.https://www.mapa.gob.es/es/alimenta cion/temas/produccion-eco/caracterizaciondecompradoresecologicosencanalespecializa dojul17_tcm30-419446.pdf 
4. Este estudio ha sido realizado por AIJU (Instituto Tecnológico de Producto Infantil y Ocio) para la AEFJ que es la Asociación Española de Fabricantes de Juguetes. AIJU (2020, noviembre) Investigación Sostenibilidad y consumo de juguetes en España. AEFJ.

5. Martin Raymond (2010) habla, entre otros métodos y técnicas habituales en el análisis de tendencias, del análisis transcultural para referirse a la observación de las diferentes culturas y sectores industriales con el objetivo de hallar los indicios de una tendencia.

6. Kids Trends Book es un cuaderno de tendencias especializado en el entorno infantil, que tiene el objetivo de ofrecer inspiración a creativos, empresas y negocios orientados al mundo de la infancia. El cuaderno propone 4 tendencias en 129 páginas con más de 450 imágenes. Cada tendencia incluye la Evolución, Indicadores, Iconos y Señales como: Colorama, Mi escenario, Juguetes, Gráfica, Materiales y Estilos de vida. El cuaderno está basado principalmente en la observación llevada a cabo en puntos de venta emblemáticos, estilos de vida, bibliografía, prensa especializada, eventos y ferias internacionales de alto impacto en el contexto del diseño infantil. El sistema de vigilancia contempla también la actualización de canales de información on-line y productos susceptibles de ser tendencia. Medio. http://nexusdesigncentre.upv.es/kidstrendsbook/

7. Esta tendencia nos describe las inquietudes de un colectivo sensible a la creatividad artística en un entorno libre y apacible donde imaginar mundos afectivos y acogedores.

8. Es un espacio estimulante de diversión científica donde se experimentan las reacciones mas locas. Niños y niñas que disfrutan con la ciencia mas traviesa y sorprendente.

9. Plantea un contexto donde el poder y la fuerza dominan la escena. La tendencia recoge un cúmulo de sensaciones publicadas en streaming y sin filtros donde la tecnología tiene mucho que jugar.

10. En este caso se aportan las claves de un mundo en transformación que se moviliza para asegurar un futuro sano. La sostenibilidad comprometida con cuidarnos y cuidar el entorno.

11. La creciente iniciativa Fridays for Future (FFF) es un movimiento global iniciado en el contexto estudiantil que reclama una acción inmediata para frenar el cambio climático y el calentamiento global. La iniciativa se plantea desde el colectivo infantil/juvenil que son los habitantes del planeta que heredarán de forma inminente, un planeta devastado por las consecuencias del cambio climático. Medio. https://fridaysforfuture.org

12. El programa Boîte à Lunch fue iniciado por la Table de Concertation Jeunesse NDG en 2003. Este programa de educación alimentaria incluye actividades, campamentos de día, talleres gratuitos de cocina y nutrición después de la escuela para niños y adolescentes con el objetivo de fortalecer la seguridad alimentaria de los jóvenes. Medio.https://boitealunch.org/

13. Peggy Notebaert Nature Museum y The Chicago Academy of Sciences proponen actividades para conectar a los ciudadanos con la naturaleza y la ciencia. En el museo se pueden ver animales nativos vivos y docenas de especies de mariposas exóticas; conocer el arte inspirado en la naturaleza o disfrutar de espacios de juego con el objetivo de entretener; educar y sensibilizar a los visitantes en el respeto por la naturaleza y el valor de la ciencia. Medio. https://naturemuseum.org/

14. Surfers Against Sewage es una ONG de conservación marina que inspira, une y capacita a las comunidades para que tomen medidas para proteger los océanos, las playas, los 
ríos y la vida silvestre. Su proyecto Ocean Guardians, pretende sensibilizar los más pequeños en el cuidado y mantenimiento de las playas y océanos para asegurar unas aguas libres de contaminación. Medio. https://www.sas.org.uk/news/ocean-plastic-emer gency-kits/ 15. Human Rights Reads for Kids. Es una iniciativa de Unicef para el Día de los Derechos Humanos donde se leen la 'Convención sobre los Derechos del Niño' que explica quiénes son los niños, cuáles son sus derechos y cuáles las responsabilidades de los gobiernos. Medio. https://www.unicef.org/es/convencion-derechos-nino/convencion-version-ninos 16. Greta Thunberg es activista ambiental conocida por su campaña de lucha contra el cambio climático. En 2018 comenzó una protesta climática en solitario a la que se unieron decenas de miles de estudiantes escolares y universitarios de todo el mundo. Una huelga climática que se ha convertido en un acontecimiento regular (Fridays For Future) respaldado por niñxs y jóvenes de todo el planeta. Medio. https://www.instagram.com/ gretathunberg/?igshid=oubjx $96 \mathrm{t} 7 \mathrm{t} 1 \mathrm{n}$

17. Chloé Sucrée es influencer en redes sociales y embajadora del batchcooking como filosofía de vida familiar. Es autora de libros sobre organización en la alimentación saludable. Medio. https://www.beingbiotiful.com/

18. Malala Yousafzai es activista de derechos humanos conocida por luchar para que las niñas en su país puedan tener acceso a una educación gratuita y obligatoria. En 2017 fue nombrada Mensajera de la Paz de las Naciones Unidas para ayudar a sensibilizar acerca de la importancia de la educación de las niñas. Medio. https://malala.org/

19. Fionn Ferreira es un joven científico e ingeniero preocupado por encontrar formas de combatir los niveles de contaminación plástica en los océanos. En 2019 fue premiado en la Feria de Ciencia de Google por su método para extraer micro-plásticos del agua. Medio. https://www.fionnferreira.com/

20. Mari Copeny, es activista de derechos sociales conocida por la defensa de su comunidad en una devastadora crisis de agua tóxica en su ciudad y la recaudación de fondos para apoyar a los niñxs desfavorecidos en todo el país. Medio. https://www.maricopeny.com/

21. Nido Muebles es una firma de diseño de mobiliario infantil centrada en la incorporación del trabajo artesanal local en el diseño y la fabricación de sus piezas. Cuenta con una línea de muebles infantiles y accesorios cargada de sensibilidad en la que la seguridad, la función y el juego tienen un papel fundamental. La colección 'Traven', diseñada por Christian Vivanco, debe su nombre al escritor Bruno Traven por su cuento 'Canastitas en serie' el cual hace referencia a la relación entre artesanos y la industria del consumo. Medio. https://nidomuebles.com

22. Residuo cero y máxima calidad es la premisa de Plan Toys que ofrece juguetes ecológicos, sostenibles y respetuosos con los niños, las niñas y el planeta. 'Wonky Fruits' es un juguete para cortar frutas y verduras con formas imperfectas. Ayuda a los niños y niñas a comprender que los beneficios de las frutas y verduras son los mismos independientemente de su aspecto, con el objetivo de reducir el desperdicio de alimentos. Medio. https:// www.plantoys.com

23. Bobo Choses es una marca de moda infantil preocupada por el mundo, que trabaja con fabricantes locales. La creatividad, la honestidad, la pasión y el sentido del humor son los valores fundamentales de la firma, que diseña ropa divertida con patrones cómodos que permiten a los niños y niñas moverse con facilidad, jugar y divertirse. La firma escribe 
libros infantiles ilustrados que inspiran sus colecciones. Medio. https://www.bobochoses. com

24. Little Eco Baby es un servicio de venta, alquiler y lavandería de pañales de tela, respetuosos con el medio ambiente. La marca propone un cambio en la vida cotidiana de las familias que supone una verdadera reducción del impacto en el medio ambiente a la vez que evita el contacto de la piel de los bebés con los productos químicos utilizados en los pañales desechables. Medio. https://ittleecobaby.com.au

25. El diseño etnográfico estudia un grupo determinado de personas a través de métodos de recogida de datos como la observación.

26. Henrik Vejlgaard es un sociólogo de las tendencias que planteó en 2008 el 'Modelo de Diamante de las Tendencias'. En su planteamiento categoriza diferentes niveles de introducción de las tendencias en la sociedad, siendo los 'creadores de tendencias' los pioneros e introductores de estas. Vejlgaard, H. (2008). Anatomía de una tendencia. Mc Graw-Hill interamericana.

27. Producto 1: Tahuna, diseñado por Inés Lorenzo y Reem Hosn.

28. Producto 2: Aywa, diseñado por Elena Fortes.

29. Producto 3: Green City, diseñado por Lamis Fawaz.

30. Munari, B. (1981) ¿Cómo nacen los objetos? Apuntes para una metodología proyectual. Editorial Gustavo Gili.

31. Desde el diseño existen infinidad de métodos de investigación centrados en la innovación y en los usuarios. Bella Martin y Bruce Hanington en su libro 'Universal Methods of Design' describen 100 métodos para desarrollar ideas innovadoras a través de soluciones efectivas de diseño.

32. Son especialmente referencia para este estudio metodologías como el Design Thinking, el Benchmarking o los Customer Journey recogidas en: Brown, T. (2008). Design Thinking. Harvard Bussines Review. Medio. https://dschool.stanford.edu

\section{Referencias}

AIJU (2020, noviembre). Investigación Sostenibilidad y consumo de juguetes en España. AEFJ. Brown, T. (2008). Design Thinking. Harvard Bussines Review. Medio. https://dschool. stanford.edu

Martin, B. y Hanington, B. (2012). Universal Methods of Design. Rockport Publishers, Beverly.

Ministerio de Agricultura y Pesca, Alimentación y Medio Ambiente (2017, julio). Caracterización de compradores de productos ecológicos en canal especializado. Secretaría General Técnica Centro de Publicaciones. Medio. https:/www.mapa.gob.es/es/alimentacion/ temas/produccion-eco/caracterizaciondecompradoresecologicosencanalespecializado jul17_tcm30-419446.pdf

Munari, B. (1981). ¿Cómo nacen los objetos? Apuntes para una metodología proyectual. Editorial Gustavo Gili.

Raymond, M. (2010). Tendencias: qué son, cómo identificarlas, en qué fijarnos. Promopress. Vejlgaard, H. (2008). Anatomía de una tendencia. Mc Graw-Hill interamericana. 
V.V.A.A. (2020). Kids Trends Book 2022. NEXUS Design Centre.

V.V.A.A. (2020, 23 de febrero). Surveys of participants in Fridays For Future climate protests on 20-28 September, 2019, in 19 cities around the world. Medio. https://osf.io/asruw/

World Commission on Environment and Development (1987). Our Common Future: Brundtland Report. Oxford University Press.

\begin{abstract}
The growing concern about climate change in children's audiences, manifested through global activism, generates a demand that designers must respond to. The study presents a tool for creatives, based on trend analysis, which allows the development of new sustainable products. The tool is based on the observation of lifestyles, business cases and design trends whose methodology provides keys to propose innovative briefings for sustainable products. A visual code instrument that offers information on consumer behavior and allows to know in advance products and aesthetics that will be in force facilitating a strategic direction for design.
\end{abstract}

Keywords: childhood - child - activism - trend - sustainable - observation - design - innovation - strategy.

Resumo: A crescente preocupação com as alterações climáticas no público infantil, manifestada através do activismo global, gera uma procura à qual os designers devem responder. O estudo apresenta uma ferramenta para os criadores, baseada na análise de tendências, que permite o desenvolvimento de novos produtos sustentáveis.

A ferramenta baseia-se na observação de estilos de vida, casos de empresas e tendências de design cuja metodologia fornece as chaves para propor briefings inovadores sobre produtos sustentáveis. Uma ferramenta de código visual que fornece informações sobre o comportamento do consumidor e permite conhecer antecipadamente os produtos e a estética que estarão em vigor. Facilitar uma direcção estratégica do design.

Palavras chave: crianças - activismo - tendência - sustentável - observação - design - inovação - estratégia.

[Las traducciones de los abstracts fueron supervisadas por el autor de cada artículo] 